\title{
Lung retransplantation: walking a thin line between hope and false expectations
}

\author{
Laurent Godinas $^{1,2}$, Dirk Van Raemdonck ${ }^{1,3}$, Laurens J. Ceulemans ${ }^{1,3}$, Robin $\operatorname{Vos}^{1,2}$, Geert M. Verleden ${ }^{1,2}$ \\ ${ }^{1}$ Lung Transplantation Unit, ${ }^{2}$ Department of Respiratory Disease, ${ }^{3}$ Department of Thoracic Surgery, University Hospitals Leuven, Leuven, Belgium \\ Correspondence to: Prof. Geert M. Verleden, MD, PhD, FERS. Lung Transplantation Unit, University Hospitals Leuven, 49, Herestraat, 3000 \\ Leuven, Belgium. Email: geert.verleden@uzleuven.be. \\ Provenance: This is an invited article commissioned by the Academic Editor Xianglin Hu (Department of Pulmonary Medicine, Zhongshan Hospital, \\ Fudan University, Shanghai, China). \\ Comment on: Wallinder A, Danielsson C, Magnusson J, et al. Outcomes and Long-term Survival After Pulmonary Retransplantation: A Single-Center \\ Experience. Ann Thorac Surg 2019;108:1037-44.
}

Submitted Sep 22, 2019. Accepted for publication Oct 31, 2019.

doi: $10.21037 /$ jtd.2019.11.02

View this article at: http://dx.doi.org/10.21037/jtd.2019.11.02

Lung transplantation is a recognized treatment for endstage lung disease for selected patients for whom no other options are available (1). Since the introduction of calcineurin inhibitors in the 1980's, lung transplantation became a reasonable treatment with improved outcomes (2). However, chronic rejection or chronic lung allograft dysfunction (CLAD) remains the major limitation for long-term survival $(3,4)$, accounting for more than $40 \%$ of deaths beyond the first year after pulmonary transplantation (4). CLAD leads to a progressive and irreversible loss of function of the transplanted organ. Two important phenotypes have been characterized: Bronchiolitis obliterans Syndrome (BOS), which was already described early in the history of thoracic transplantation (5) and the first guidelines for diagnosis and staging were published in 1993 (3). In 2011, Sato et al. reported another form of CLAD, known as restrictive allograft syndrome (RAS) (6). During the last decades research has led to a better understanding of the pathophysiological mechanisms leading to CLAD. The classification, the diagnostic criteria and the management of CLAD were recently updated, illustrating the advances in the field (4). Although some medical treatments or other strategies have potentially demonstrated usefulness either to prevent (for instance azithromycin) or to stabilize CLAD (extracorporeal photophoresis, total lymphoid irradiation) (4), BOS and RAS remain impossible to cure by using these interventions. In general pulmonary function decline is progressive despite all interventions, leading to increased symptoms and disability, chronic respiratory failure and finally death. Therefore, redo transplantation has so far been considered as the only possible treatment option in a selected cohort of these desperate patients $(7,8)$. Concomitantly, ethical issues arose, related to redo transplantation with potentially worse outcomes in an era of shortage of donor lungs (9).

In this issue of The Annals of Thoracic Surgery, Wallinder and co-authors (10) compared the outcomes of 49 retransplanted patients with 635 primarily transplanted patients. In their study, the global population of retransplanted patients displayed significantly worse outcomes at 30 days, 1-, 2- and 5-year with a survival of respectively $90 \%, 76 \%, 71 \%$ and $55 \%$, compared to $94 \%$, $82 \%, 76 \%$ and $61 \%$ in their 635 primary transplanted patients. However, after adjustment for confounding factors, such as the time period after surgery and retransplantation within the first year, the statistical significance disappeared. This, again, clearly illustrates that early retransplantation (i.e., within the first year after transplantation) presents a high risk of mortality. However, in the remaining cohort, retransplantation for CLAD resulted in similar outcomes compared to a first transplantation.

Although encouraging, this data deserves a word of caution. Retransplantation has been introduced since the end of the 1980's with an improvement of the mortality thanks to the growing experience of the lung transplant centers $(2,11)$. The registry of the International Society 
for Heart and Lung Transplantations (ISHLT) reported that redo transplantation constitutes about $5 \%$ of the annual transplant procedures (2). However, in the ISHLT multicenter cohort, the retransplanted patients had a survival at 3 months and 1 year of $91 \%$ and $83 \%$ respectively, and a median survival of 6.3 years in the most recent era, which is still worse compared to primary transplantation (2). The discrepancy of survival between Wallinder et al. (10) and the ISHLT data is probably due to a highly selected population that underwent a strict screening process, excluding patients with comorbidities and frailty markers.

Factors influencing lower survival of retransplanted patients were evaluated in a previous large study by Kawut et al. (11). They also found that early retransplantation was a risk factor for poor survival after redo transplantation, independent of many other recipient or procedure characteristics. After adjustment, mechanical ventilation did not emerge as a risk factor for death (11), in line with other studies (12) and comparable to the results of Wallinder et al. (10) who found that bridging to transplantation with extracorporeal membrane oxygenation or mechanical ventilation was not an independent risk factors of mortality. Interestingly, Kawut et al. also demonstrated that risk of BOS recurrence was higher in patients retransplanted for CLAD accounting for a substantial part of the mortality (11). Wallinder and co-authors did not specifically assess this in their study.

Other authors already clearly demonstrated that early retransplantation leads to poor survival $(11,13)$. This suggests that redo lung transplantation should be predominantly reserved for patients with CLAD and not for patients suffering from suture stricture/dehiscence or severe primary graft dysfunction (PGD). Indeed, several other studies have specifically assessed the role of retransplantation as the ultimate treatment for CLAD patients. Although retransplantation for CLAD was acceptable in terms of outcomes with 1 and 5 years survival of respectively $89.2 \%$ and $64.3 \%$ which was not different compared to primary transplantation, CLAD retransplanted patients presented more peri-operative complications, such as severe PGD, need for peri-operative extracorporeal membrane oxygenation and bleeding (14). Another study, evaluating specifically outcomes of retransplanted CLAD patients, displayed a survival rate for BOS retransplanted patients of $60 \%, 53 \%$ and $45 \%$ at respectively 1,3 and 5 years with a quite high mortality of $30 \%$ in the first 6 months after transplantation, as a result of early complications such as graft infection and PGD (12). Osaki et al. found a survival rate for CLAD patients at 1, 2 and 5 years of respectively $67 \%, 67 \%$ and $44 \%$, which was lower than their primary transplanted patients (12). It seems indeed that experience of the center is extremely important to select the patients for redo transplantation and for sure to do the retransplantation itself. This was clearly illustrated in the multicenter study by Verleden et al. showing that the phenotype of CLAD also impacts on the survival after redo lung transplantation (15). Indeed, they have shown that patients presenting with RAS not only have a worse survival but also a lower CLAD-free survival after redo compared to patients transplanted for BOS. This worse outcome was in part driven by a high early post-operative mortality but also by an increased recurrence of CLAD. Early post-operative mortality in those patients could be due to the complexity of the surgical procedure and more specifically the increased risk of bleeding in patients with extensive pleural adhesions, worse condition prior to transplantation due to severe hypoxic respiratory failure and more frequent requirement of bridge to transplantation with extracorporeal membrane oxygenation. However, after adjusting the statistical analysis taking into account the above mentioned parameters, RAS remained an independent predictor of poor survival after redo transplant (15).

The relapse of CLAD in this particular redo population is not clearly understood, but may be due to the persistence of the immunological substrate responsible for the pathological process, such as persistence of (subclinical) antibody mediated rejection (16), or psychosocial factors such as medication non-adherence (17). Similar observations were recently published in another study which showed lower survival in patients retransplanted for RAS than for BOS, although the low number of patients preclude definitive conclusions but reinforce previous results (18). Therefore, selection of the candidates for retransplantation should take into account the chance of success for patients according to the current phenotype of CLAD. Wallinder et al. (10) have not looked into the potential differences in survival between CLAD phenotypes, which is a kind of a missed opportunity to either corroborate or to invalidate existing results.

Although retransplantation is performed in most of the lung transplantation centers with acceptable outcomes as Wallinder and colleague have again demonstrated in this issue of The Annals of Thoracic Surgery (10), the indication for redo lung transplantation should be always carefully weighted against the risks of such a procedure, 
taking into account the ethical issues of donor shortage. Retransplantation for early graft dysfunction or suture problems within the first year should be discouraged. Retransplantation remains, however, a reasonable option for patients with CLAD with particular attention to the phenotype of CLAD, RAS being the worse indication with the highest risk of peri-operative complications, recurrence of the disease and long-term poorer outcomes. In lung transplantation, careful selection of the patients accounts for an important part of the success of the procedure. In view of retransplantation, the screening process has to be even more strictly performed, allowing only a minority of patients a second chance without compromising patients who are waiting for their first transplantation.

Most of the patients presenting with CLAD will ask about the possibility to receive a second chance, perceiving the retransplantation as the only hope to prolong their life, sometimes with unrealistic expectation. However, regarding the poor outcomes in some subsets of patients, the risk of recurrent CLAD and the shortage of available lung, retransplantation should remain restricted to a very selected lung transplant population. The ethical issue to perform redo or not will commonly arise in the discussion with the CLAD patient, leading the lung transplant physician to frequently refuse the patient request and to draw a line between hope and false expectations, between what is reasonable and what is unrealistic.

\section{Acknowledgments}

None.

\section{Footnote}

Conflicts of Interest: The authors have no conflicts of interest to declare.

Ethical Statement: The authors are accountable for all aspects of the work in ensuring that questions related to the accuracy or integrity of any part of the work are appropriately investigated and resolved.

\section{References}

1. Weill D, Benden C, Corris PA, et al. A consensus document for the selection of lung transplant candidates: 2014--an update from the Pulmonary Transplantation Council of the International Society for Heart and Lung
Transplantation. J Heart Lung Transplant 2015;34:1-15.

2. Yusen RD, Edwards LB, Kucheryavaya AY, et al. The registry of the International Society for Heart and Lung Transplantation: thirty-first adult lung and heart-lung transplant report--2014; focus theme: retransplantation. J Heart Lung Transplant 2014;33:1009-24.

3. Cooper JD, Billingham M, Egan T, et al. A working formulation for the standardization of nomenclature and for clinical staging of chronic dysfunction in lung allografts. International Society for Heart and Lung Transplantation. J Heart Lung Transplant 1993;12:713-6.

4. Verleden GM, Glanville AR, Lease ED, et al. Chronic lung allograft dysfunction: Definition, diagnostic criteria, and approaches to treatment-A consensus report from the Pulmonary Council of the ISHLT. J Heart Lung Transplant 2019;38:493-503.

5. Burke CM, Theodore J, Dawkins KD, et al. Posttransplant obliterative bronchiolitis and other late lung sequelae in human heart-lung transplantation. Chest 1984;86:824-9.

6. Sato M, Waddell TK, Wagnetz U, et al. Restrictive allograft syndrome (RAS): a novel form of chronic lung allograft dysfunction. J Heart Lung Transplant 2011;30:735-42.

7. Miller JD, Patterson GA. Retransplantation following isolated lung transplantation. Semin Thorac Cardiovasc Surg 1992;4:122-5.

8. Fournier M, Sleiman C, Mal H, et al. Single-lung retransplantation for late graft failure. Eur Respir J 1993;6:1202-6.

9. Estenne M, Knoop C. Lung retransplantation. Eur Respir J 1993;6:1093-4.

10. Wallinder A, Danielsson C, Magnusson J, et al. Outcomes and Long-Term Survival after Pulmonary Retrasplantation A single Center Experience. Ann Thorac Surg 2019;108:1037-44.

11. Kawut SM, Lederer DJ, Keshavjee S, et al. Outcomes after lung retransplantation in the modern era. Am J Respir Crit Care Med 2008;177:114-20.

12. Brugière $\mathrm{O}$, Thabut $\mathrm{G}$, Castier $\mathrm{Y}$, et al. Lung retransplantation for bronchiolitis obliterans syndrome: long-term follow-up in a series of 15 recipients. Chest 2003;123:1832-7.

13. Osaki S, Maloney JD, Meyer KC, et al. Redo lung transplantation for acute and chronic lung allograft failure: long-term follow-up in a single center. Eur J Cardiothorac Surg 2008;34:1191-7.

14. Biswas Roy S, Panchanathan R, Walia R, et al. Lung 
Retransplantation for Chronic Rejection: A Single-Center Experience. Ann Thorac Surg 2018;105:221-7.

15. Verleden SE, Todd JL, Sato M, et al. Impact of CLAD Phenotype on Survival After Lung Retransplantation: A Multicenter Study. Am J Transplant 2015;15:2223-30.

16. Verleden SE, Vanaudenaerde BM, Emonds MP, et al. Donor-specific and -nonspecific HLA antibodies and outcome post lung transplantation. Eur Respir J 2017;50. doi: 10.1183/13993003.01248-2017.

17. Dew MA, DiMartini AF, Dobbels F, et al. The 2018

Cite this article as: Godinas L, Van Raemdonck D, Ceulemans LJ, Vos R, Verleden GM. Lung retransplantation: walking a thin line between hope and false expectations. J Thorac Dis 2019;11(11):E200-E203. doi: 10.21037/jtd.2019.11.02
ISHLT/APM/AST/ICCAC/STSW Recommendations for the Psychosocial Evaluation of Adult Cardiothoracic Transplant Candidates and Candidates for Longterm Mechanical Circulatory Support. Psychosomatics 2018;59:415-40.

18. Revilla-López E, Berastegui C, Sáez-Giménez B, et al. Lung Retransplantation Due to Chronic Lung Allograph Dysfunction: Results From a Spanish Transplant Unit. Arch Bronconeumol 2019;55:134-8. 